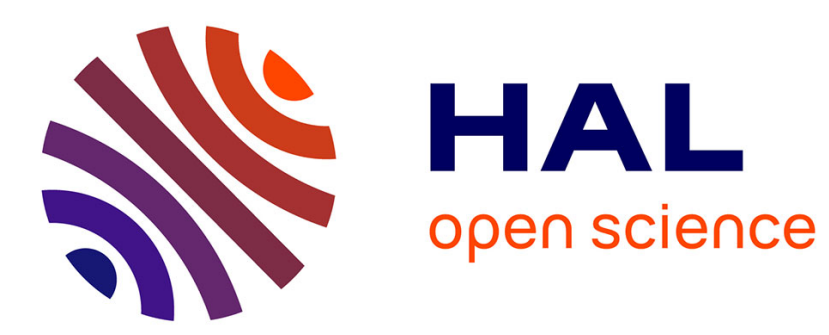

\title{
ANTENNES À DIAGRAMME DE DIRECTIVITÉ PARTICULIERS OBTENUS PAR PONDÉRATION SPATIALE
}

\author{
C. Thirard, J. Pasqualini
}

\section{- To cite this version:}

C. Thirard, J. Pasqualini. ANTENNES À DIAGRAMME DE DIRECTIVITÉ PARTICULIERS OBTENUS PAR PONDÉRATION SPATIALE. Journal de Physique IV Proceedings, 1992, 02 (C1), pp.C1-993-C1-997. 10.1051/jp4:19921218 . jpa-00251185

HAL Id: jpa-00251185

https://hal.science/jpa-00251185

Submitted on 1 Jan 1992

HAL is a multi-disciplinary open access archive for the deposit and dissemination of scientific research documents, whether they are published or not. The documents may come from teaching and research institutions in France or abroad, or from public or private research centers.
L'archive ouverte pluridisciplinaire HAL, est destinée au dépôt et à la diffusion de documents scientifiques de niveau recherche, publiés ou non, émanant des établissements d'enseignement et de recherche français ou étrangers, des laboratoires publics ou privés. 


\title{
ANTENNES À DIAGRAMME DE DIRECTIVITE PARTICULLERS ORTENUS PAR PONDERATION SPATIALE
}

\author{
C. THIRARD et J.P. PASQUALINI* \\ METRAVIB R.D.S., 64 Chemin des Mouilles, BP. 182, F-69132 Ecully cedex, France \\ "CERDAN, D.C.N. Toulon, BP. 77, F-83800 Toulon-Naval, France
}

\section{Bésumé :}

L'utilisation classique d'une antenne acoustique conduit à des diagrammes de directivité qui évoluent avec la fréquence. Par pondération spatiale de l'antenne, on peut obtenir des lobes principaux à caractéristiques constantes sur une large bande. Trois techniques de calcul des coefficients de pondération sont proposées qui permettent d'obtenir des diagrammes de directivité (1) à résolution constante (2) hyper-directifs (3) en forme de créneau.

\section{Abstract:}

Acoustic arrays have usually radiation patterns which are not constant when the frequency varies. By multiplicating each sensor with a coefficient, we can obtain radiation patterns with a constant main beamwidth over a large frequency band. Three computation techniques of the coefficients are proposed : the first one produces radiation patterns with constant resolution, the second produces superdirective radiation patterns and the last produces radiation patterns with rectangular shape.

\section{INTRODUCTION -}

L'utilisation classique d'une antenne acoustique -préformation dans une direction donnée par rotation électronique- conduit à des diagrammes de directivité qui évoluent avec la fréquence. Celte caractéristique est pénalisante lorsque, par exemple, l'on cherche à estimer zône par zône le rayonnement d'un bruiteur étendu. En effet, non seulement la taille de la zône observée, mais également le nombre de zónes nécessaires pour recouvrir le bruiteur dépend de la fréquence.

Afin de s'affranchir de cet inconvénient on utilise une méthode de pondération spatiale de l'antenne qui conduit à des lobes principaux avec des caractéristiques invariantes sur une large bande. On présente trois techniques de calcul permettant d'obtenir, pour chaque fréquence, les coefficients de pondération correspondants. Suivant la technique utilisée, on obtient un diagramme de directivité à résolution constante (\$II), hyper-directif (\$III) ou en forme de créneau (\$IV).

\section{DIAGRAMME DE DIRECTIVITE A RESOLUTION CONSTANTE -}

L'idée de base est d'utiliser une fenêtre de pondération spatiale paramètrée : à chaque fréquence, le choix du paramètre permet d'imposer une contrainte au diagramme de directivité. Le diagramme de directivité de l'antenne pondérée, à la fréquence $f$, s'exprime par :

$$
D_{\alpha}(\theta, f)=\left[\sum_{i=1}^{N} g_{\alpha}\left(x_{i}\right) e^{-j k_{o}\left(\sin \theta-\sin \theta_{0}\right) x_{i}}\right]\left[\sum_{i=1}^{N} g_{\alpha}\left(x_{i}\right)\right]-1
$$

oủ $N$ est le nombre d'hydrophones de l'antenne, $k_{0}=2 \pi f / c_{0}$ est le nombre d'ondes acoustiques, $\theta_{0}$ est la direction de préformation, $x_{i}$ repère la position du jème hydrophone par rapport au centre de l'antenne et $g_{\alpha}(x)$ est la fenêtre de pondération paramétrée par $\alpha$. Soit $f_{m}$ la fréquence minimale de la bande 
considérée et $\theta_{r}$ l'angle vérifiant $D_{0}\left(\theta_{r}, f_{m}\right)=0,5$ où $D_{0}(\theta, f)$ est le diagramme non pondéré : on choisit à la fréquence $f$ le paramètre $\alpha(f)$ afin que lon ait $D_{\alpha}(f)\left(\theta_{r}, f\right)=0,5$. On impose ainsi sur la bande de fréquence une résolution constante, égale à celle observée à la fréquence minimale : celle-ci étant la plus importante, on dégrade donc le lobe principal en l'élargissant aux fréquences supérieures. Cinq fenêtres ont été testées : $\cos ^{\alpha}$, Kaiser-Bessel, Cauchy, Gauss, Poisson. On a retenu celle de KaiserBessel, caractérisée par :

$$
g_{\alpha}\left(x_{i}\right)=I_{0}\left(\pi \alpha \sqrt{\left.1-4 x_{i}^{2} / L^{2}\right)} / I_{0}(\pi \alpha)\right.
$$

où lo est la fonction de Bessel modifiée de première espéce d'ordre 0 et $L$ est la longueur de l'antenne. En effet, c'est la fenêtre qui réduit le plus le niveau des lobes secondaires du diagramme.

La figure 1 présente les diagrammes obtenus avec cette fenêtre, une antenne a cinq hydrophones préformée dans la direction $\theta_{0}=0^{\circ}$ et sur une octave caractérisée par $\Delta x / \lambda=0,52$ à la fréquence maximale, si $\Delta x$ est la distance entre deux hydrophones consécutifs et $\lambda$ la longueur doonde.

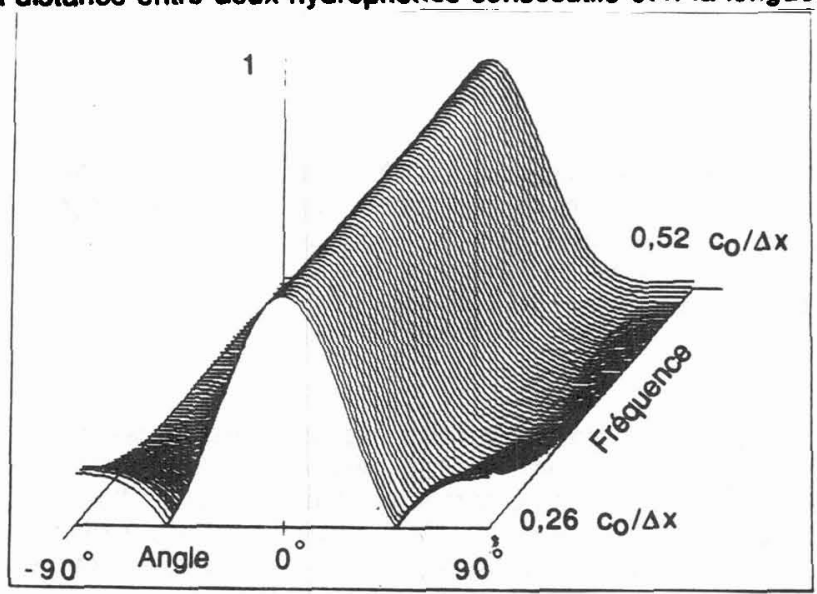

Elqure nْ1 - Diagrammes de directivité sur une octave - Antenne à 5 hydrophones pondérée par fenêtre de Kaiser-Bessel.

\section{DIAGRAMME DE DIRECTIVITE HYPER-DIRECTIE -}

L'objectif de cette pondération est de pouvoir figer la résolution du diagramme de directivité sur une bande de fréquence, en obtenant, pour les fréquences les plus faibles, un pouvoir séparateur supérieur à celui correspondant à une antenne non pondérée. Deux méthodes ont été développées par DUHAMEL /1/ et DAWOUD et ANDERSON /2/ dans le cas d'antennes a $\mathrm{N}=2 \mathrm{M}+1$ hydrophones. En utilisant des coefficients de pondération pairs, on peut mettre le diagramme de directivité de l'antenne préformée dans la direction $\theta_{0}=0^{\circ}$ sous la forme :

$$
D(\theta, f)=\sum_{i=0}^{M} \varepsilon_{i} a_{i} \cos (i \psi)
$$

où $\varepsilon_{0}=1, \varepsilon_{i}=2$ si $i \neq 0$ et $\psi=k_{0} \Delta x \sin \theta$. Les fonctions $\cos (i \psi)$ s'exprimant en fonction des puissances de cos $\psi, D(\theta, f)$ est égal à $P_{M}(\cos \psi)$ où $P_{M}$ est un polynôme de degré $M$ dont les coefficients dépendent des a. DUHAMEL identifie alors $P_{M}(\cos \psi)$ au polynôme $T_{M}$ de Tchebyschev de degré $M$, agissant sur la variable

$$
z=\frac{z_{0}+1}{1-\cos \left(k_{0} \Delta x\right)} \cos \psi-\frac{z_{0} \cos \left(k_{0} \Delta x\right)+1}{1-\cos \left(k_{0} \Delta x\right)}
$$


On constate que lorsque $\theta$ varie de $0^{\circ}$ à $90^{\circ}, z$ varie de $z_{0}$ a $-1: z_{0}$ permet donc de fixer le rapport entre le niveau du lobe principal et celui des lobes secondaires qui est égal à $1 / T_{M}\left(z_{0}\right)$ (figure 2). On obtient un diagramme de directivité avec des lobes secondaires de méme niveau, ce niveau étant minimal pour une résolution donnée (également fixée par $z_{0}$ ).

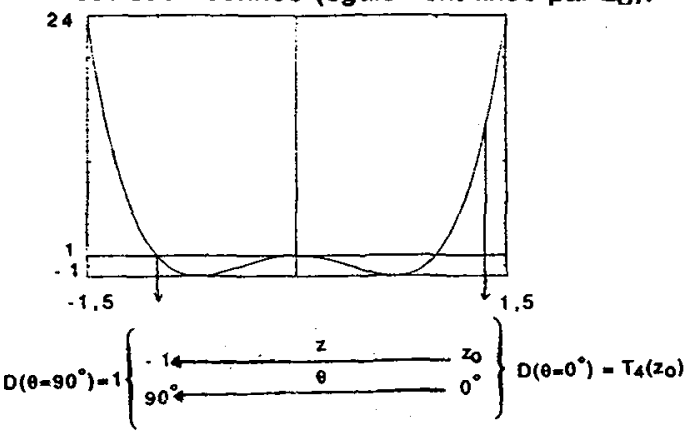

Eiqure $n^{\circ} 2$ - Parcours sur le polynöme de Tchebyschev $\mathrm{T}_{4}$ de la variable $\mathrm{z}$ quand $\theta$ varie de 0 à $90^{\circ}$.

Le calcul des aj s'effectue à partir de l'égalité $P_{M}(\cos \psi)=T_{M}(z)$. L'inconvénient de la méthode de DUHAMEL est qu'elle conduit à des diagrammes de directivité peu robustes : une erreur de $1 \%$ sur un coefficient conduit a un diagramme complètement déformé. L'idée de DAWOUD est de substituer a TM un polynôme mieux adapté $Q_{M}$ qui a une pente plus forte pour $z \geq 1$ et des oscillations plus faibles lorsque

$0 \leq z \leq 1$.

II propose d'utiliser le polynóme $T_{M}-T_{M-1}$ pour lequel on augmente légèrement les coefficients de plus fort degré : par exemple, pour $M=4$, nous avons utilisé $Q_{4}(z)=10 z^{4}-3 z^{3}-8 z^{2}+3 z+1$. On obtient les nouveaux coefficients de pondération en égalant $P_{M}(\cos \psi)$ et $Q_{M}(z)-a$ où

$$
z=\frac{z_{0}-z_{1}}{1-\cos \left(k_{0} \Delta x\right)} \cos \psi-\frac{z_{0} \cos \left(k_{0} \Delta x\right)-z_{1}}{1-\cos \left(k_{0} \Delta x\right)}
$$

Comme précédemment, $z_{0}$ permet de fixer le niveau des lobes secondaires et $z_{1}$ est la valeur prise par $z$ quand $\theta=90^{\circ}$; a est une translation sur $Q_{M}$ permettant de symmétriser par rapport à 0 les extrema présentés par $Q_{M}$ dans l'intervalle $\left[z_{0}, 2_{1}\right]$ (figure3). La figure 4 montre les diagrammes de directivité obtenus par ces deux méthodes avec une antenne de 9 capteurs et $k_{0} \Delta x=90^{\circ}$.

$$
6.5
$$

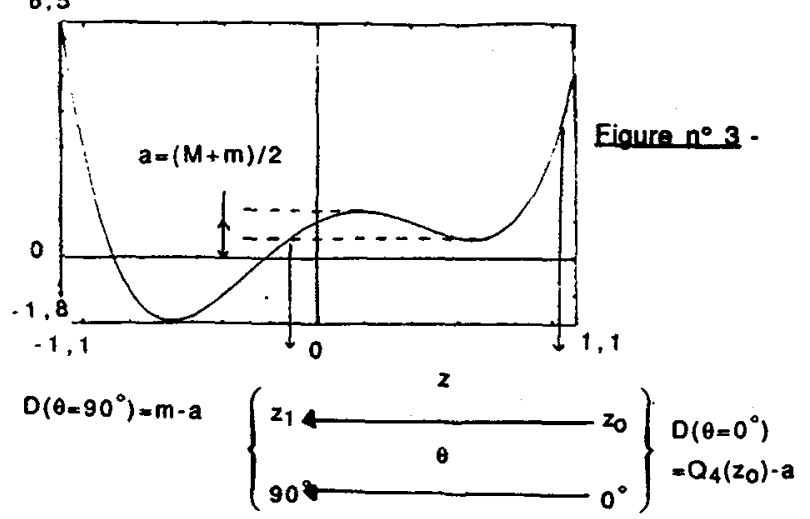

Eioure $n^{\circ} 3$ - Parcours sur le polynome de $Q_{4}$ de la variable $z$ quand $\theta$ varie de 0 a $90^{\circ}$.

Eloure n०4 - Diagrammes de directivité $N=9, k_{0} \Delta x=90^{\circ}$. O: sans pondération -1 : DUHAMEL - 2 : DAWOUD

\section{Eiaure $n^{\circ} 4$.}

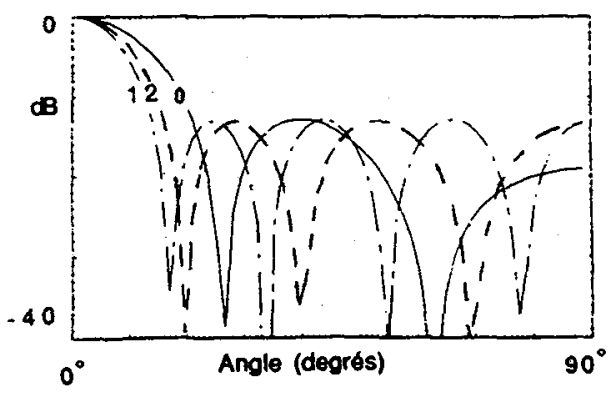




\section{Cas d'utilisation : aide au déconfinement -}

Si l'on est dans une configuration confinée pour laquelle une source image se trouve sur une direction située dans le lobe principal du diagramme de directivité de l'antenne non pondérée, on se rapprochera de la configuration champ libre en utilisant un diagramme hyperdirectif. L'idéal est alors de faire coîncider la direction de la source image avec le $1^{\mathrm{er}}$ zéro du diagramme de l'antenne pondérée.

\section{DIAGRAMME DE DIRECTIVITE EN FORME DE CRENEAU -}

On cherche à obtenir un diagramme de directivité dont le module soit le plus proche possible d'un gabarit $\mathrm{G}(\theta)$ caractérisé par une bande passante (valeur 1) entourée de deux bandes atténuées (valeur 0 ). Le gabarit considéré est invariant sur une large bande de fréquence.

Pour une fréquence donnée $f, D(\theta, f)$ est la transformée de Fourier de la réponse impulsionnelle $\left(a_{i}\right) i=1, N$ calculée pour la variable $k=k_{0} \sin \theta$ :

$$
D(\theta, f)=\sum_{i=1}^{N} a_{j} e^{-j k_{0}} \sin \theta x_{i}
$$

La détermination des coefficients de pondération (aj) se ramène alors à un problème classique de synthèse de filtre si l'on substitue au gabarit en $\theta$ le gabarit en nombre d'onde $k$ défini par :

$$
H_{f}(k)=G\left(\arcsin \left(k / k_{0}\right)\right) \text { si } k \leq k_{0}, H f(k)=0 \text { si } k>k_{0} .
$$

Pour calculer les aj, on utilise alors l'algorithme de REMEZ $/ 3 /$ qui, pour une erreur maximale $\delta_{1}$ autorisée dans la bande passante et une erreur maximale $\delta_{2}$ dans les bandes atténuées, fournit la fonction de transfert avec la plus faible largeur de transition. II est possible, en gardant la même valeur $\delta_{1}$ quelque soit la fréquence d'ajuster la variable $\delta_{2}$ afin d'obtenir des diagrammes de directivité qui aient le méme comportement dans la bande de transition. De tels diagrammes sont illustrés figure 5 , pour une antenne à 27 capteurs et pour 3 fréquences dans l'octave $0,25 c_{0} / \Delta x-0,5 c_{0} / \Delta x$.

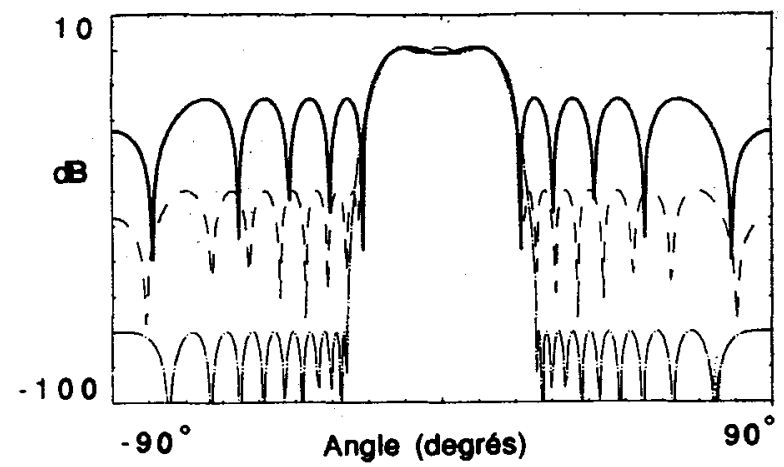

Elaure 5 - Diagrammes de directivité en créneau

$$
\begin{aligned}
& -f=0,25 c_{0} / \Delta x \\
& --f=0,375 c_{0} / \Delta x \\
& -\cdot-f=0,5 c_{0} / \Delta x
\end{aligned}
$$

\section{v. CONCLUSIONS -}

Trois techniques de pondération spatiale d'antenne ont été présentées. La première conduit à des diagrammes ayant une largeur de lobe principal constante sur une large bande ; on pourrait lui substituer une autre contrainte comme, par exemple, la position du ler zéro. La deuxième permet d'améliorer la résolution de l'antenne et également de fixer la position du 1er zéro du diagramme. La dernière méthode permet de synthétiser un diagramme de directivité dont la forme est donnée et est indépendante de la fréquence. 
Ces techniques sont aisées à mettre en oeuvre, soit dans le domaine fréquentiel -en pondérant l'antenne à chaque fréquence avec les coefficients adéquats- soit dans le domaine temporel en utilisant pour chaque capteur un convolueur numérique dont la fonction de transfert représente les variations du coefficient de pondération du capteur dans la bande de fréquence considérée.

BEMERCIEMENTS : Les auteurs remercient le CERDAN qui a financé ces travaux.

\section{BIBLIOGRAPHIE -}

$11 /$ DUHAMEL, R.H., Proceedings of the I.R.E., Oct.1952

121 DAWOUD, M.M. et ANDERSON, A.P., Design of superdirective arrays with high radiation efficiency, I.E.E.E. Transactions, Vol. AP-26, ${ }^{\circ} 6$, Nov. 1978

/ 3 / RABINER, L.R. et GOLD, B. Theory and application of digital signal processing. PRENTICE-HALL 